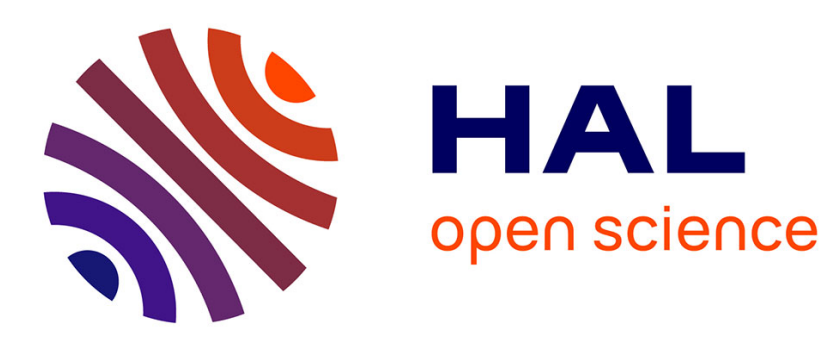

\title{
Les limites du territoire: une application de la notion de rayon de confiance au développement territorial
}

\author{
J.M. Callois
}

\section{To cite this version:}

J.M. Callois. Les limites du territoire: une application de la notion de rayon de confiance au développement territorial. Revue d'économie régionale et urbaine, 2007, 5, p. 811 - p. 830 . hal-00455633

\section{HAL Id: hal-00455633 \\ https://hal.science/hal-00455633}

Submitted on 10 Feb 2010

HAL is a multi-disciplinary open access archive for the deposit and dissemination of scientific research documents, whether they are published or not. The documents may come from teaching and research institutions in France or abroad, or from public or private research centers.
L'archive ouverte pluridisciplinaire HAL, est destinée au dépôt et à la diffusion de documents scientifiques de niveau recherche, publiés ou non, émanant des établissements d'enseignement et de recherche français ou étrangers, des laboratoires publics ou privés. 
JOBNAME: No Job Name PAGE: 1 SESS: 15 OUTPUT: Mon Dec 17 15:57:17 2007 /juridique/acolin-RERU/444863m/05/01_callois

Version auteur de Revue d'Economie Régionale et Urbaine, 2007, 5, 811-830

http://reru.u-bordeaux4.fr/

http://www.cairn.info

\title{
Les limites du territoire :
}

Une application de la notion de rayon de confiance au développement territorial *

\section{The limits of the territory :}

\section{An application of the notion of "radius of trust" to territorial development}

\author{
Jean-Marc CALLOIS \\ Ingénieur-chercheur \\ Unité mixte de recherches METAFORT (Cemagref-ENGREF-ENITA-INRA) \\ Équipe " économie des espaces ruraux " \\ 24, avenue des Landais \\ BP 50085 \\ 63172 Aubiere Cedex \\ jean-marc.callois@clermont.cemagref.fr
}

Mots clés : développement territorial, rayon de confiance, capital social, études de cas.

Keywords : territorial development, radius of trust, social capital, case studies.

Classification JEL : R11, R12.

* Première version mars 2006, version révisée juin 2007

L'auteur remercie Antoine SOUBEYRAN pour ses conseils sur le développement de la notion de rayon de confiance, ainsi que les deux rapporteurs qui ont grandement aidé à clarifier et à rendre plus rigoureuse la partie théorique. Merci également à Serge HeRviou pour la réalisation des figures et de la carte. 
Les limites du territoire

\section{Résumé}

L'approche dite territoriale du développement régional consiste à supposer que certaines zones peuvent être considérées comme des systèmes auto-organisés d'acteurs, et que les caractéristiques socioculturelles sont des déterminants essentiels des dynamiques de développement. Cet article vise à évaluer le domaine de validité de l'approche territoriale en proposant un modèle théorique qui détermine les conditions d'organisation endogène d'un territoire, et en présentant les résultats de deux études de cas sur des zones rurales françaises. Les résultats suggèrent que l'approche territoriale devrait être réservée aux zones caractérisées par une cohésion élevée et homogène dans l'espace et entre les secteurs économiques.

\section{Summary}

The so-called territorial approach in regional development consists in assuming that some areas may be considered as self-organised systems of actors, and that socio-cultural features are essential determinants of development dynamics. This article aims at evaluating the domain of validity of the territorial approach. It first provides a theoretical model of endogenous organisation of a territory, and then presents the results of two case studies on French rural areas. These results suggest that a territorial approach should be limited to the areas characterised by a high and homogenous cohesion, both in spatial terms and across economic sectors.

\section{Introduction}

L'approche territoriale jouit d'une certaine popularité auprès des théoriciens comme des praticiens du développement régional. Du côté des théoriciens, plusieurs programmes de recherche mettent en avant l'intérêt de considérer de petites régions comme des entités organisées, voire " organiques », et de mettre l'accent sur les potentialités d'organisation endogène de ces zones. Il s'agit notamment de la littérature sur les districts industriels (COURLET, 2001), sur « l'économie des proximités » (PECQueur et Zimmerman, 2004) ou sur les « milieux innovateurs » (MAILlat et al., 1991). Du côté des praticiens, on note une tendance croissante à mettre l'accent sur la "territorialisation des politiques publiques» comme une façon d'adapter au mieux l'intervention publique aux besoins locaux (CÉLIMÈNE, 1999 ; MAHÉ, 2002). Des dispositifs récents, comme la réforme de l'intercommunalité en France et surtout la politique des pays ( $c f$. infra), ont généré quantité de diagnostics et de stratégies de développement basés sur une approche territoriale.

L'approche territoriale, envisagée comme approche de recherche, sera définie ici par la réunion de deux hypothèses. Premièrement, elle suppose l'existence de territoires, qui doivent être appréhendés comme des systèmes auto-organisés d'acteurs, et dans lesquels les dimensions économique, socioculturelle et institutionnelle sont étroitement liées et « font système ». L'analyse d'un territoire doit être intégrée et non sectorielle, car l'étude séparée des différents secteurs d'activités ne permet pas la compréhension des mécanismes de développement à l'œuvre. En effet, et c'est là la deuxième hypothèse, ces mécanismes de développement dépendent dans une large mesure de facteurs socioculturels spécifiques à chaque territoire.

Plusieurs auteurs mettent en doute la pertinence d'un recours exagéré à l'approche territoriale. DION et LACOUR (2000) plaident pour une attention plus marquée 
aux caractéristiques des différents secteurs économiques, et plus généralement aux forces économiques classiques. De façon plus radicale, AMIN (1993) dénonce le "mythe» du retour aux économies régionales. Selon lui, à côté des quelques exemples emblématiques qui ont donné naissance à la vogue du développement "par le bas" (LAMARChE, 1995), la désintégration des économies régionales se poursuit inlassablement.

Le but de cet article est de contribuer à évaluer la pertinence d'une approche territoriale dans l'analyse du développement régional. Pour cela, il procède en deux étapes. Tout d'abord, à partir de la notion de rayon de confiance introduite par FuKUYAMA (1995) au niveau international, il propose une analyse théorique de la mobilisation collective spontanée d'individus situés géographiquement. Elle met en évidence certaines conditions pour qu'une organisation stable puisse émerger sur une zone donnée. Ces conditions sont mises en regard de données empiriques sur la cohésion locale. Ensuite, deux études de cas sont présentées, pour évaluer l'existence d'effets proprement territoriaux. Les deux zones d'étude ont été choisies de manière à présenter des divergences importantes en matière de dynamisme économique, qui pourraient s'interpréter comme la conséquence de caractéristiques territoriales différentes. En réalité, les résultats suggèrent que non seulement le rôle des facteurs territoriaux spécifiques est marginal dans ces deux territoires, et en outre qu'aucun de ces territoires ne peut être considéré comme un système auto-organisé d'acteurs. Les implications de ces résultats en matière de politiques territoriales sont discutées en conclusion.

\section{-1 - \\ Sous quelles conditions une " cohésion territoriale " peut-elle émerger ?}

Une hypothèse cruciale de l'approche territoriale en matière de développement régional est qu'il est possible d'identifier des zones géographiques (les "territoires ») sur lesquelles une organisation endogène se met en place pour favoriser le développement. Or, rien n'assure qu'une telle organisation puisse émerger effectivement d'elle-même.

Dans cette section, on analyse de manière théorique sous quelles conditions des " groupes de cohésion » peuvent se constituer de manière endogène, et donc donner un sens à la notion de territoire comme organisation émergeant des acteurs locaux eux-mêmes (et non pas impulsée de l'extérieur). Pour cela, un modèle heuristique simple est proposé, qui permet de déterminer les conditions sous lesquelles une organisation endogène se constitue ${ }^{1}$.

\subsection{Un modèle fondé sur le rayon de confiance}

On considère un espace abstrait qui englobe différentes notions de proximité (géographique, culturelle etc.). Les agents sont répartis sur un segment $[0, N]$ où ils forment un continuum de densité uniforme ${ }^{2}$. Une telle représentation est pertinente pour les productions dont les acteurs sont atomistiques et dispersés dans 
Les limites du territoire

l'espace (par exemple des prestataires touristiques ou des producteurs de biens agricoles de qualité, souvent visés par les politiques territoriales et les recherches sur le développement territorial). Ce type de représentation peut également convenir pour les districts industriels.

On suppose qu'il n'existe qu'un seul bien, produit avec rendements croissants : de la sorte, l'action collective est profitable. Ces rendements croissants peuvent correspondre à divers avantages que peut conférer la coordination et l'organisation collective : prospection commune de marchés, politique de communication, acquisition d'un pouvoir de marché, biens publics locaux, résolution de problèmes d'externalités etc. On supposera qu'il n'y a pas de limite aux économies d'échelle, de sorte que les agents ont toujours intérêt à intégrer le plus grand groupe possible.

La représentation des relations de coopération se fonde sur la notion de rayon de confiance $^{3}$, introduite par Francis FuKUYAMA (1995). On suppose que chaque agent est caractérisé par son " rayon de confiance ». La figure 1 illustre une «fonction de confiance » possible (l'axe horizontal représente l'espace, et l'axe vertical le rayon de confiance). Un agent positionné en $x$, au rayon de confiance $r(x)$ fera confiance à tous les agents du segment $[x-r(x), x+r(x)]$, mais à aucun agent extérieur à ce segment ${ }^{4}$. Si le segment $[x-r(x), x+r(x)]$ dépasse les limites de l'espace $[0, N]$, on ne retient bien entendu que la partie où sont localisés les agents, $c^{\prime}$ est-à-dire $[x-r$ $(x), x+r(x)] \cap[0, N]$.

Cette représentation implique que la relation de confiance n'est pas nécessairement réciproque. Pour prendre un exemple trivial, un habitant d'un bourg pourra avoir un espace d'identification plus vaste qu'un habitant d'un village isolé, dont l'espace de référence pourra se restreindre à son hameau. La notion de confiance est donc très liée aux représentations des agents. Deux agents peuvent se faire confiance a priori sans se connaître. La notion de rayon de confiance traduit donc aussi la prédisposition à travailler ensemble. Plutôt que de rayon de confiance, on pourrait également parler d'ouverture d'esprit, ce qui explique mieux pourquoi la confiance peut ne pas être réciproque. L'élément essentiel à retenir est que la proximité géographique n'implique par nécessairement la capacité à travailler collectivement, et que c'est de cette variabilité spatiale dans la capacité d'action collective que va découler l'émergence des territoires.

La fonction de confiance est exogène, supposée être le produit de diverses évolutions historiques et contraintes géographiques. Bien que ce caractère exogène soit critiquable, l'essentiel pour notre propos est que le rayon de confiance soit suffisamment stable dans le temps à l'échelle de la mise en place des politiques et stratégies territoriales. On supposera en outre la fonction de confiance dérivable. En revanche, l'organisation des agents est endogène et détermine l'apparition de " groupes de cohésion » cohérents, qui seront assimilés aux territoires. Un groupe de cohésion est défini comme un ensemble d'agents au sein duquel tous les individus se font mutuellement confiance. Formellement : $|y-x|$

$G$ est un groupe de cohésion si et seulement si $\forall x, y \in G, r(x) \geq|y-x|$ et $r(y) \geq$ 


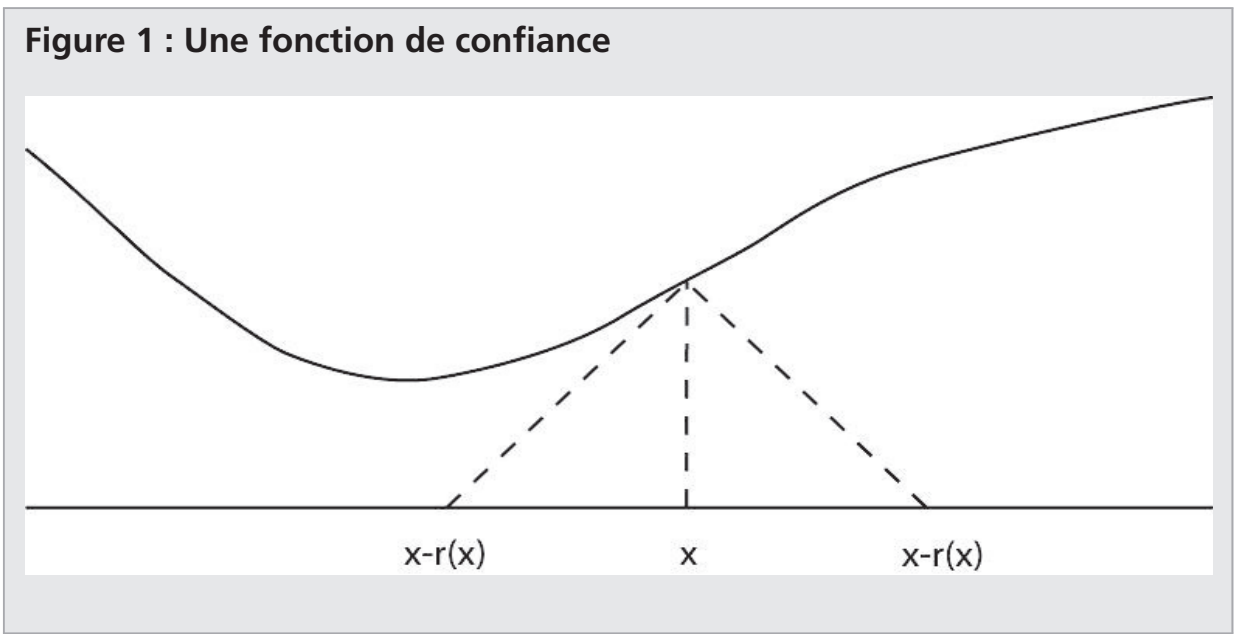

Il s'agit donc de déterminer une segmentation stable de l'espace en groupes de cohésion.

\subsection{Détermination des groupes de cohésion maximaux}

On commence par étudier les groupes de cohésion maximaux (par rapport à la relation d'inclusion des ensembles), c'est-à-dire des groupes de cohésion où il n'est pas possible d'agréger un individu extérieur au groupe sans que la propriété de cohésion soit perdue, autrement dit : $H$

G est maximal si et seulement si pour tout groupe de cohésion $H, G \subset H \Rightarrow G=$

On peut alors montrer le résultat suivant (la preuve est donnée en annexe 1) :

\section{Proposition 1}

Supposons que $\left|r^{\prime}(x)\right|<1$ pour tout $\mathrm{x}$, que et que, alors :

- Tous les groupes de cohésion maximaux sont d'un seul tenant (connexes).

- Les groupes de cohésion maximaux sont des segments $[a, b]$ de longueur $b-a=\min \{r(a), r(b)\}$.

- L'ensemble des groupes de cohésion maximaux est l'ensemble des intervalles $[a, a+r(a)]$ tels que $r(a) \leq r(a+r(a))$, et des intervalles $[b-r(b), b]$ tels que $r$ $(b) \leq r(b-r(b))$.

Ainsi, dans le cas où la fonction de confiance varie de façon modérée dans l'espace, les groupes de cohésion maximaux possèdent de «bonnes propriétés »: ils ne sont pas "mités» (i.e. ils sont d'un seul tenant), et les valeurs du rayon de 
Les limites du territoire

confiance à leur bordure déterminent leur taille. Les schémas de la figure 2 illustrent quelques configurations possibles. La partie en gras indique le groupe de cohésion maximal. Les lignes diagonales sont des droites à $45^{\circ}$ délimitant le domaine de confiance d'un individu. On note que c'est toujours la valeur de bord la plus petite qui détermine la taille du groupe (et non la valeur minimale de la fonction comme on aurait pu le penser). En pratique, la proposition 1 et la figure 2 signifient que lorsque la portée des relations sociales (rayon de confiance) est relativement homogène, la taille d'un territoire est déterminée par les propriétés sociologiques à la frontière du territoire (et non par celles de l'intérieur). Pour avoir un territoire vaste (donc des coopérations à grande échelle), il faut que les conditions y soient propices partout y compris (voire surtout) sur les bords. Une application concrète de ce résultat se retrouve lorsque l'on observe la structuration des projets de Pays autour des agglomérations. Dans certains cas, on observe la constitution d'un "grand pays » incluant l'agglomération, dans d'autres une "marguerite» de petits pays périurbains. En général, cette dernière configuration provient de l'attitude défensive des zones périurbaines. Ce sont elles qu'il faudrait «travailler » pour élargir les coopérations.

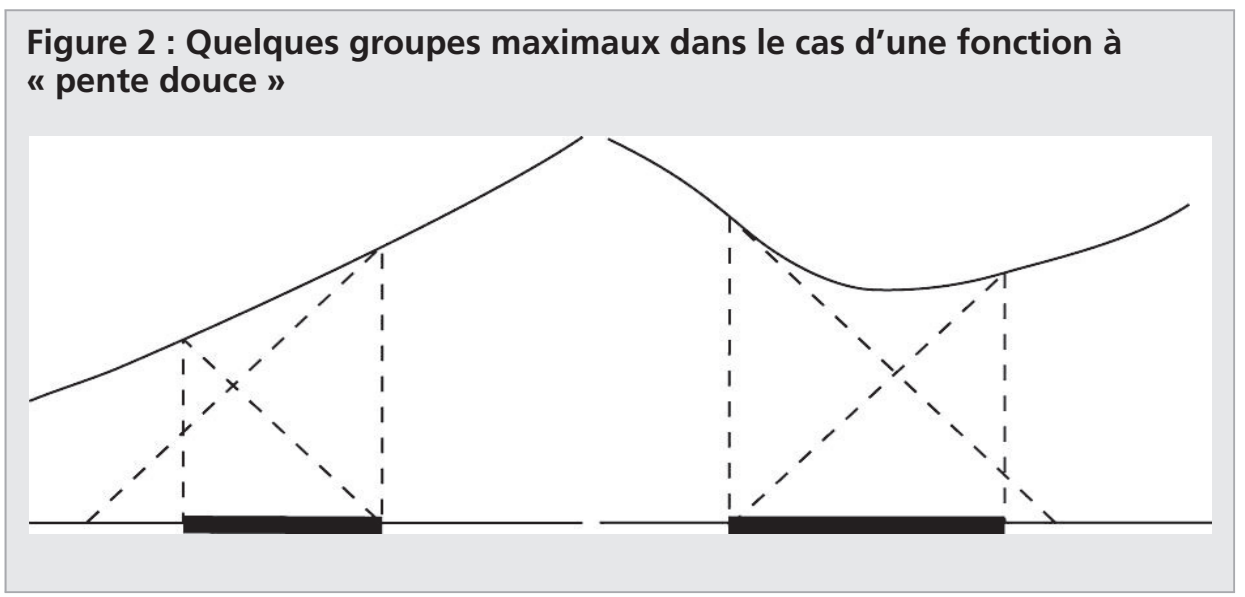

En revanche, comme l'illustre la figure 3, si la fonction de confiance connaît des variations abruptes, les groupes de cohésion peuvent présenter des propriétés très différentes. En particulier, les valeurs intérieures de la fonction de confiance peuvent contraindre leur taille (fig. 3, à gauche), et les groupes maximaux peuvent désormais être « mités " (fig. 3, à droite).

Deux enseignements importants ressortent de cette analyse. Premièrement, la moyenne seule du rayon de confiance sur une zone ne suffit pas à déterminer l'ampleur de la captation des économies d'échelle. Si les variations du rayon de confiance sont importantes dans l'espace, il peut y avoir de très petits groupes qui se constituent ( $\mathrm{d}^{\prime}$ où une faible efficacité), malgré une moyenne de fonction de confiance qui peut être élevée. Deuxièmement, l'échelle pertinente d'observation de l'effet de la cohésion locale peut se situer à un échelon géographique assez fin pour comprendre le fonctionnement d'un territoire. 


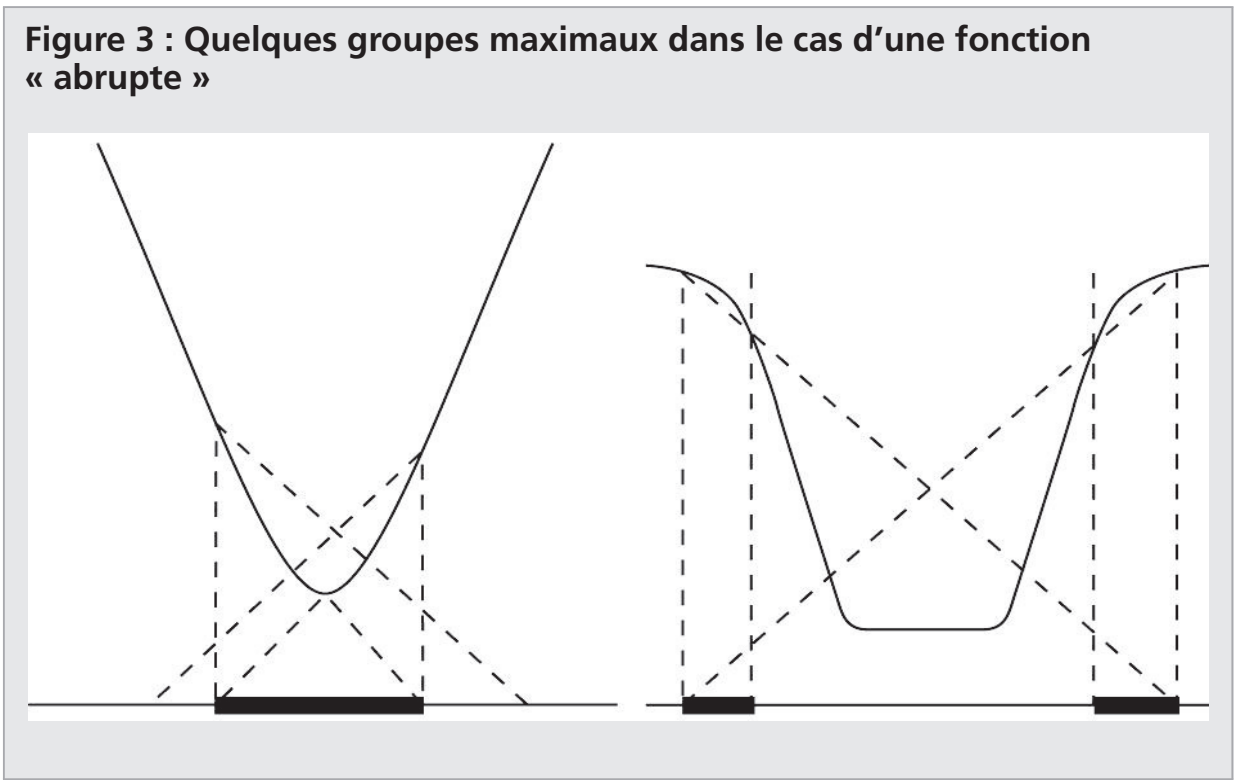

\section{3. Équilibre spatial}

L'objectif de ce modèle est d'étudier comment (sans être données a priori) des territoires peuvent émerger à partir des caractéristiques des individus. Pour cela, il est nécessaire de définir une notion d'équilibre spatial. On définira ici un équilibre spatial comme une partition de l'espace en groupes de cohésion dans laquelle aucun ensemble d'agents n'a intérêt à constituer un nouveau groupe de cohésion, sachant qu'un agent appartient au plus à un seul groupe de cohésion. Formellement :

$\left\{G_{1}, \ldots G_{n}\right\}$ est un équilibre si et seulement si :

a) $\left\{G_{1}, \ldots G_{n}\right\}$ est une partition de $[0, N]$

b) $\forall i \in\{1,2 \ldots, n\}, G_{i}$ est un groupe de cohésion

c) $\forall H \subset[0 ; N]$ tel que $H$ est un groupe de cohésion, $\exists x \in H$ tel que $\|H\| \leq\left\|G_{i(x)}\right\|$ où $i(x)$ est l'unique indice $i(x) \in\{1,2 \ldots, n\}$ tel que $x \in G_{i(x)}$, et où $\|$.$\| désigne la mesure$ usuelle dans \&\#8476;.

À l'équilibre, tout l'espace est recouvert par des groupes de cohésion (sans recouvrement entre groupes), et aucun groupe d'agents ne peut décider de former un groupe déviant sans que l'un au moins des agents voie sa situation se dégrader ou rester stable (en d'autres termes, il n'est pas possible que tous les agents d'un tel groupe gagnent à dévier) ${ }^{5}$. On a alors le résultat suivant, dont la preuve est donnée en annexe 2 :

\section{Proposition 2}

Pour toute fonction de confiance $r$ telle que, au moins un équilibre existe. 
Les limites du territoire

L'existence d'un équilibre est un résultat rassurant du point de vue de la validité de l'approche territoriale. Cependant, le processus de constitution des équilibres spatiaux peut aboutir à des situations assez surprenantes, et sensibles à de petites modifications de la fonction de confiance. Il se peut notamment que des groupes très petits se constituent malgré un rayon de confiance assez élevé, situation qui peut être à la fois considérée comme inefficace (on n'exploite pas tout le potentiel d'économies d'échelle) et inéquitable. La figure 4 donne un exemple d'une telle situation. Sur ce schéma, les groupes 1 et 3 se constituent en premier, laissant aux agents localisés au milieu la possibilité de former le groupe 2, qui paraît bien petit par rapport à la valeur du rayon de confiance de ses agents. En effet, chacun de ses agents a un rayon de confiance plus élevé que la taille du groupe 2, et même plus élevé que celui de la plupart des membres des deux autres groupes! Notons qu'une telle situation, qui paraît assez "inéquitable », peut émerger que la fonction de confiance connaisse des variations abruptes ou non.

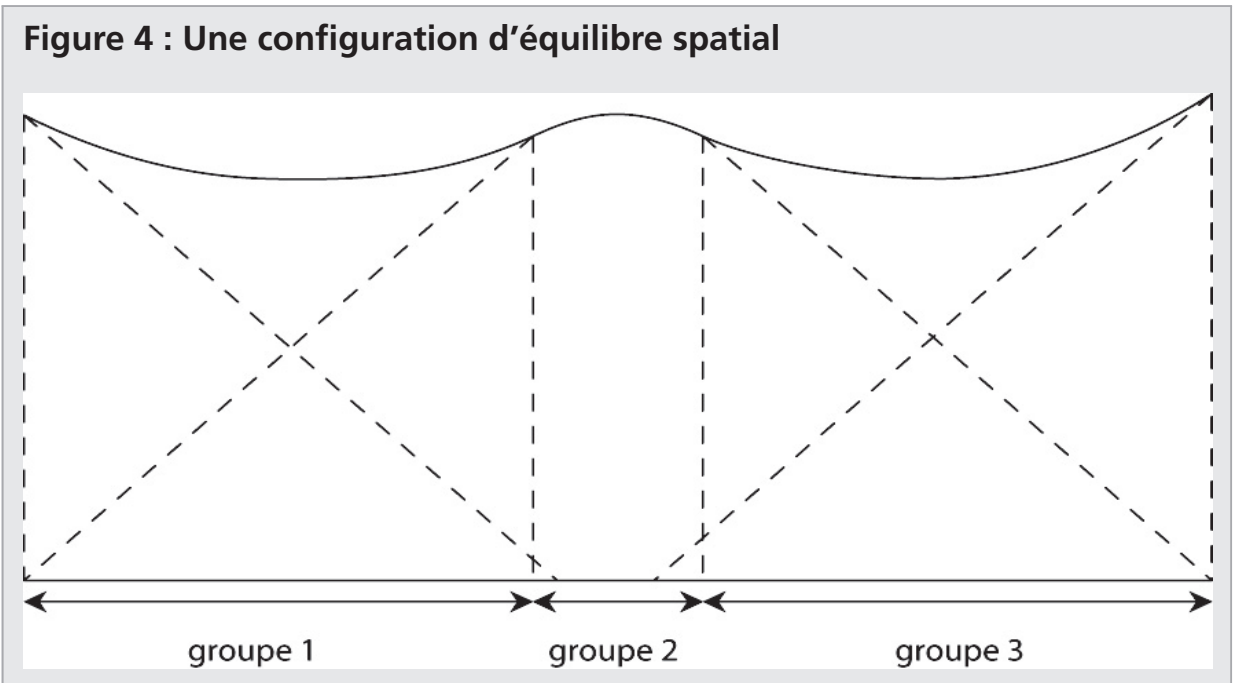

Dans ce dernier cas, il est peu probable que l'approche " ascendante " se révèle satisfaisante du point de vue d'un planificateur, et une intervention correctrice pourra s'avérer souhaitable. En effet, il paraît injuste qu'une zone bénéficie d'économies d'échelle très inférieures à ce que son niveau de rayon de confiance minimal pourrait permettre.

Ce modèle pointe donc deux difficultés potentielles à l'approche territoriale entendue comme capacité d'organisation endogène. D'abord, en cas de variations brutales du rayon de confiance, des configurations insatisfaisantes (mitées, trop petites) peuvent émerger. Ensuite, la répartition spatiale endogène des « territoires » peut aboutir à des potentialités inexploitées en matière d'action collective. 


\subsection{Retour au réel}

Dans la réalité, en dehors de la nécessité d'adopter un cadre bidimensionnel, divers facteurs peuvent nuancer ces résultats. Par exemple, si l'on applique ce modèle à la mise en cuvre d'une politique touristique, il est évident que le territoire sur lequel la politique sera appliquée devra correspondre à une zone cohérente en termes de patrimoine naturel et culturel. Toutefois, il est probable que ces caractéristiques seront cohérentes avec la fonction de confiance. En effet, plusieurs auteurs insistent sur le fait que les caractéristiques sociologiques liées à la capacité d'action collective sont déterminées en grande partie par des facteurs géographiques et culturels profonds, qui évoluent très lentement dans le temps (PuTNAM, 1993; TODD, 1990), ce qui justifie de les considérer comme exogènes. L'émergence concrète de l'action collective dépendra cependant des opportunités présentes et du niveau d'économie d'échelles. Dans le cadre de problématiques liées au développement rural, une organisation spatiale efficace est typiquement considérée comme essentielle dans le cas du tourisme, de l'agriculture (coopération, signes de qualité...), et des services à la population. Le fait que les zones rurales présentent des différences assez tranchées en matière de capacité d'organisation collective (CALLOIS, 2004), suggère que la capacité d'organisation collective dépend en partie de facteurs sociologiques locaux, assimilables à la notion de rayon de confiance.

Pour aller plus loin dans la mise à l'épreuve empirique des résultats de cette section, des études de terrain s'avèrent nécessaires, car il est très délicat de mesurer la notion de rayon de confiance à l'aide d'indicateurs statistiques. En première approximation, il est toutefois possible d'utiliser des indicateurs de cohésion sociale locale, tels que celui élaboré par CALLOIS et AUBERT (2007) ${ }^{6}$. Un tel indicateur est cependant très imparfait, car il est tout à fait possible d'avoir une forte cohésion mais un faible rayon de confiance (le cas du pays de Saint-Flour discuté dans CALLOIS et AUBERT (2007) est très éloquent à cet égard).

La répartition spatiale de l'indicateur de cohésion locale pour les zones rurales françaises est reproduite en annexe 3. Malgré une forte autocorrélation spatiale positive, il existe des zones où l'hétérogénéité spatiale de l'indicateur de cohésion est forte. Ces zones permettent de tester indirectement les résultats de cette section, qui suggèrent que l'émergence spontanée de territoires d'une taille importante est peu probable lorsque le rayon de confiance présente une grande hétérogénéité spatiale. C'est l'objet de la section suivante, qui se propose de confronter au terrain une implication centrale du modèle : le rôle du rayon de confiance et de l'hétérogénéité des caractéristiques sociologiques sur l'extension des coopérations et actions collectives.

\section{-2 - \\ Deux études de cas}

Le but de cette section est d'examiner la validité des deux hypothèses fondant l'approche territoriale : l'existence de systèmes auto-organisés d'acteurs et le rôle des facteurs socioculturels spécifiques aux territoires dans leur développement. Elle 
Les limites du territoire

constitue également un test indirect des résultats de la section précédente, qui suggèrent que l'approche territoriale est peu appropriée lorsque les facteurs permettant la cohésion présentent une grande hétérogénéité spatiale. Pour cela, deux territoires qui présentent une évolution économique contrastée sont étudiés.

\subsection{Le choix des territoires}

Les zones d'études choisies sont des pays au sens de la Loi d'Orientation pour le Développement Durable du Territoire (LOADDT) de 1999. La loi définit les pays comme des territoires présentant " une cohésion géographique, culturelle, économique ou sociale» (article 22). Un pays est « reconnu, à l'initiative des communes ou de leurs groupements " (article 22) mais doit impliquer, outre les élus locaux, l'ensemble des acteurs concernés par le développement économique, social, culturel, et environnemental. Reconnu par l'État, le pays devient un espace privilégié pour mettre en ouvre une stratégie de développement et obtenir des financements publics. La politique des pays découle donc directement de la «philosophie » de l'approche territoriale ${ }^{7}$.

Les deux zones d'étude sont les pays des Combrailles et Sancerre-Sologne ( $c f$. carte en annexe 3). Ces zones présentent une certaine hétérogénéité au niveau de l'indicateur de cohésion, et sont donc " peu exemplaires " au regard du modèle idéal du territoire de projet sociologiquement homogène, mais ce sont a contrario des zones adaptées à une confrontation empirique du modèle de la section I. Elles ont aussi été choisies de manière à avoir des caractéristiques relativement semblables en matière de dotations en facteurs et accessibilité. En revanche, elles diffèrent nettement par leur performance économique. Il s'agit alors de déterminer si des facteurs non marchands et en particulier les caractéristiques socioculturelles peuvent expliquer ces différences.

Le tableau 1 présente quelques caractéristiques des deux terrains choisis. On note plusieurs points communs en matière de facteurs " classiques » de développement : - Les deux pays sont assez vastes, mais peu denses, et ont une superficie et une population du même ordre de grandeur.

- Tous deux sont bordés par des voies de communication importantes mais non traversées par elles. Bien qu'étant situés en quasi-totalité dans l'espace à dominante rurale, ils sont assez proches de grosses aires urbaines.

- Aucun n'a de ville centre vraiment dominante, mais tous deux ont des pôles ruraux plus ou moins importants.

- La structure économique présente un secteur primaire et une industrie toutes deux assez importantes, concentrée sur quelques pôles (malgré la fermeture des mines en Combrailles). L'activité agricole, relativement homogène en Combrailles (élevage bovin dominant) est cependant plus diversifiée en Sancerre-Sologne (zones viticoles, de grandes cultures, de polyculture-élevage). 


\begin{tabular}{|c|c|c|c|}
\hline & Combrailles & $\begin{array}{l}\text { Sancerre- } \\
\text { Sologne }\end{array}$ & $\begin{array}{l}\text { Moyenne } \\
\text { (pays } \\
\text { reconnus) }\end{array}$ \\
\hline Taille $\left(\mathbf{k m}^{2}\right)$ & 2082 & 2370 & 1258 \\
\hline Nombre de communes & 103 & 62 & 81 \\
\hline Population 1999 (milliers) & 49,4 & 58,1 & 75,5 \\
\hline Emploi 1999 (milliers) & 14,4 & 19,6 & 27,2 \\
\hline Part du secteur primaire 1999 & $20 \%$ & $15 \%$ & $11 \%$ \\
\hline Part du secteur secondaire 1999 & $26 \%$ & $27 \%$ & $22 \%$ \\
\hline Part de bacheliers 1999 & $18 \%$ & $20 \%$ & $17 \%$ \\
\hline Croissance de l'emploi 1990-99 & $-6 \%$ & $-1 \%$ & $+2 \%$ \\
\hline Croissance de la population 1990-99 & $-5 \%$ & $-1 \%$ & $+2 \%$ \\
\hline $\begin{array}{l}\text { Revenu moyen des ménages } 1999 \\
(€)\end{array}$ & 21267 & 25078 & 24719 \\
\hline Potentiel fiscal par hab. 2001 (€) & 411 & 517 & 492 \\
\hline $\begin{array}{l}\text { Coefficient d'intégration fiscale } \\
2002\end{array}$ & $15 \%$ & $2 \%$ & $22 \%$ \\
\hline $\begin{array}{l}\text { Distance moyenne à l'autoroute } \\
(\mathrm{km}) 1998\end{array}$ & 34 & 32 & 24 \\
\hline $\begin{array}{l}\text { Aire urbaine la plus proche } \\
\text { (minutes) } 1998\end{array}$ & $36^{\prime}$ & $27^{\prime}$ & $31^{\prime}$ \\
\hline
\end{tabular}

Malgré ces points communs, la situation économique de ces deux pays est remarquablement contrastée. Sur tous les indicateurs économiques globaux, le pays des Combrailles se comporte notablement moins bien que le pays SancerreSologne. Aucune explication évidente ne s'impose à l'esprit. Le pays des Combrailles a certes un relief plus accidenté que celui de Sancerre-Sologne, ce qui peut le rendre moins attractif pour l'implantation de nouvelles activités. Son climat est plus rude et les bassins industriels voisins de Clermont-Ferrand et Montluçon connaissent également un déclin industriel important. Ces facteurs ne semblent cependant pas expliquer à eux seuls une telle différence.

Pour analyser les facteurs de développement et leurs causes, en plus de l'exploitation des données statistiques disponibles, des entretiens ont été conduits sur ces deux zones d'étude pendant le printemps 2005, auprès des institutions liées au développement (organisme du pays, chambres consulaires, associations d'entreprises etc.), ainsi qu'avec un panel d'habitants appartenant à diverses catégories sociales.

\subsection{Quels « système d'acteurs " et quel rayon de confiance sur les zones d'étude?}

À première vue, l'extension spatiale assez vaste de ces deux pays semble indiquer un rayon de confiance étendu. Or, les enquêtes de terrain montrent que ces deux 
Les limites du territoire

pays sont marqués par des disparités internes assez importantes en termes de capacité d'action collective.

La situation est la plus marquée dans le pays des Combrailles, où on peut distinguer à grands traits une partie sud (sud de la rivière Sioulet, qui coule d'Ouest en Est) où la capacité d'action collective est élevée, et une partie nord où elle est plus faible. C'est au sud qu'existe l'unique association d'entrepreneurs "Club Combrailles sud » (une tentative similaire au nord ayant échoué). La capacité de mobilisation collective s'est aussi manifestée à travers l'opposition à un projet de centre d'enfouissement de déchets nucléaires au sud-est du pays. La principale ville du pays, Saint-Éloy-les-Mines, située à l'extrême nord, se distingue par son refus d'adhérer au pays et à toute structure intercommunale. Par ailleurs, la quasi-totalité des habitants interrogés confirment l'existence de cette frontière culturelle autant que géographique, l'identité étant considérée comme plus forte au sud.

Toutefois, cette opposition nord-sud n'est pas généralisable à toutes les activités. On observe ainsi une relation inverse dans le secteur agricole, où la part d'exploitations sous signe de qualité est sensiblement plus élevée au nord qu'au sud. Le secteur agricole se caractérise d'ailleurs par une faible tendance à l'action collective. Il existe quelques initiatives (pain des Combrailles, charolaise des Combrailles), mais qui concernent très peu d'exploitations (une dizaine).

Ainsi, malgré l'existence d'un pays vaste (qui s'explique historiquement par des considérations politiques locales), il ressort de l'analyse des actions collectives existantes et des entretiens réalisés auprès des habitants que le rayon de confiance est dans l'ensemble faible sur ce territoire, bien qu'il soit plus élevé dans certaines zones (notamment au sud). En cohérence avec cette observation, force est de constater que la structure d'animation du pays n'a pas réussi à concrétiser une réelle démarche collective en matière économique. Malgré la signature d'un " contrat de territoire » avec l'État en 2004, qui débloque pourtant des financements importants du Ministère de l'Industrie et des collectivités, les actions mises en ouvres gardent toujours un caractère local, et les quelques actions réellement collectives, toujours pilotées de l'extérieur, ne vont guère au-delà des diagnostics.

Le pays Sancerre-Sologne comprend trois zones géographiques contrastées. Le Sancerrois, à l'Est, est la seule partie où l'identité est décrite par les habitants comme forte. C'est aussi la partie la plus citée pour ses capacités d'action collective, essentiellement à travers l'exemple de la viticulture (entraide, coordination à l'export...), I'AOC Sancerre possédant certains traits similaires au modèle du district italien (mélange de concurrence et coopération, travail sur l'image du produit, performance à l'exportation). En outre, la principale initiative collective en matière de développement touristique, l'association "Un sourire pour vous accueillir», a émané du Sancerrois avant de diffuser vers l'ouest du pays. Le Pays Fort, au centre du pays, contraste fortement avec le Sancerrois. C'est une zone de grandes exploitations caractérisée par un fort individualisme. Enfin, la Sologne berrichonne à l'Ouest, est une région dominée par de grandes propriétés, où la forêt occupe une place importante et croissante. C'est une zone très fermée, où les activités économiques sont peu présentes. 
En cohérence avec cette hétérogénéité, il existe très peu d'actions fédératrices au niveau de l'ensemble du pays, et on observe de grandes difficultés en matière d'organisation de services publics. Une exception notable est l'ORAC (opération de restructuration de l'artisanat et du commerce), unanimement décrite comme une opération exemplaire sur l'ensemble du pays. Il s'agit cependant d'une action essentiellement impulsée de l'extérieur (par la chambre de commerce et d'industrie).

En conclusion, le pays Sancerre-Sologne, malgré sa taille qui relève d'une volonté politique du Conseil Général, s'avère être un pays remarquablement cloisonné, à faible rayon de confiance sauf dans sa partie orientale (et sur certains secteurs économiques). Les « vrais » territoires de projets sont au nombre de trois (au moins) et la transversalité est embryonnaire.

Les observations sur les deux pays sont cohérentes avec les résultats du modèle de la section précédente : la capacité d'action collective présente de grandes variations dans l'espace et entre secteurs économiques, et l'étendue spatiale des actions collectives est faible, malgré l'existence d'une structure "politique » de taille importante. Ces deux pays contredisent donc l'image d'un territoire comme une entité cohérente auto-organisée. Voyons à présent comment interpréter la divergence de dynamisme économique entre ces deux pays.

\subsection{Quel rôle de la cohésion locale dans le dynamisme économique?}

L'indicateur moyen de cohésion sur les deux pays est très différent sur les deux pays : - 0,23 pour Sancerre-Sologne et 0,24 pour les Combrailles (la moyenne nationale pour tous les pays est de - 0,26). Ce résultat est cohérent avec les enquêtes à la population, qui mettent davantage l'accent sur les solidarités et l'identité dans les Combrailles qu'en Sancerre-Sologne. Il est pour le moins troublant qui l'indicateur de cohésion suggère une tendance à la coopération plus élevée dans les Combrailles, pays le moins dynamique sur le plan économique. En fait, c'est seulement en analysant l'évolution sectorielle de l'emploi à une échelle géographique fine que l'on peut mieux comprendre les contrastes entre ces deux pays. On s'aperçoit alors que les facteurs économiques classiques (dotations en facteur, potentiel de marché, économies d'agglomération) restent les déterminants essentiels de la situation économique.

La forte baisse de l'emploi en Combrailles, relativement homogène sur le territoire, s'explique avant tout par la poursuite de la reconversion industrielle, ainsi que par la diminution de la population agricole, le tout couplé à une forte émigration des jeunes vers les grandes agglomérations. La stagnation globale du niveau d'emploi en Sancerre-Sologne masque quant à elle de fortes disparités spatiales. C'est la zone du Sancerrois qui tire vers le haut (ou plutôt vers l'équilibre) la variation globale de l'emploi, surtout le secteur agricole et agroalimentaire très dynamique, tandis que l'emploi est relativement stable dans les autres secteurs. Les autres zones enregistrent quant à eux des pertes d'emploi assez importantes dans l'industrie et le secteur agricole. 
Les limites du territoire

Pour autant, en pays Sancerre-Sologne, il est remarquable que la dynamique spatiale de l'emploi coïncide bien avec l'estimation du niveau de capital social : elle est d'autant plus forte qu'on se rapproche du Sancerrois, pays où les valeurs collectives sont les plus affirmées dans les secteurs agricole et touristique. Concernant le pays des Combrailles, il est probable que le poids des facteurs structurels sur les secteurs économiques dominants empêche de détecter un effet de la cohésion locale au niveau du développement de l'ensemble de ce territoire. Cependant, il est possible que grâce à la cohésion locale, l'arrêt de l'activité industrielle traditionnelle ait eu des effets moins délétères que dans d'autres zones rurales (les Ardennes par exemple) grâce à des grandes entreprises valorisant les qualités particulières de la main-d'œuvre, non seulement en termes de capital humain, mais aussi de valeurs sociales. Ces caractéristiques, souvent mentionnées dans les entretiens, sont néanmoins difficiles à vérifier.

Comme attendu, les effets de la cohésion locale se font surtout sentir dans le tourisme et l'agriculture. Par contre, le secteur résidentiel (commerce, logement), et l'artisanat ne semblent guère être concernés par des effets importants de la cohésion, du fait du faible degré d'économies d'échelle et de l'absence d'enjeux technologiques. En fait, le secteur du commerce et de la construction suit essentiellement l'évolution de la population. Concernant enfin l'industrie, un rôle de la cohésion peut être supposé dans les Combrailles, bien qu'il soit assez difficile à établir avec certitude.

Cependant, malgré un rôle potentiel de la cohésion locale, l'élément déterminant reste un facteur tout à fait classique en développement régional, à savoir la maîtrise des marchés et opportunités extérieures. Ainsi, en Sancerre-Sologne, la performance sur le long terme de la filière viticole repose en partie sur une bonne organisation locale, mais surtout sur une forte maîtrise des marchés d'exportation, ce qui tranche avec d'autres appellations du val de Loire. À l'inverse, le pays des Combrailles est très pénalisé par sa faible ouverture à l'extérieur.

\section{- 3 - \\ Discussion}

Les résultats, tant théoriques qu'empiriques, mettent en question l'intérêt de considérer systématiquement un territoire comme une entité organique cohérente. En effet, une organisation collective n'émerge sur une zone, assimilable à un territoire, que sous des conditions assez exigeantes, non réalisables partout. De plus, une organisation territoriale spontanée peut aboutir à des inégalités fortes entre territoires. Enfin, les phénomènes de synergie et de coopération qui sont censés justifier l'approche territoriale n'apparaissent que dans des secteurs économiques bien précis, et peuvent être largement contrebalancés par les forces économiques classiques (accès aux marchés et aux technologies notamment).

De fait, aucun des deux territoires étudiés dans cet article ne peut être considéré comme une entité organisée de manière endogène. Adopter un point de vue 
territorial dans l'étude de ces deux pays n'aide guère à la compréhension des mécanismes de développement.

Il est bien évident que l'étude de ces deux zones ne réfute pas à elle seule la pertinence de l'approche territoriale. Cependant, elle jette un doute sur sa généralité. L'approche territoriale peut par contre s'avérer tout à fait valable dans les zones relativement homogènes sur le plan des caractéristiques socioculturelles, où la tendance à l'action collective est élevée, mais aussi où des complémentarités réelles existent entre activités économiques.

Il faut aussi insister sur le fait qu'il convient de distinguer analyse et applications : ces conclusions ne remettent pas en cause la pertinence d'une approche territoriale en tant que méthode pratique de développement. Au contraire, les actions d'animation peuvent aboutir à créer sur le long terme des conditions plus propices au développement d'actions collectives, à condition que les territoires soient définis de manière adaptée, en trouvant un équilibre entre organisation endogène et directives plus centralisées. Du modèle théorique développé ici découlent des pistes pour orienter l'action publique. Dans les zones à fort rayon de confiance, la priorité devra être donnée au développement de l'ouverture à l'extérieur. À l'inverse, dans les zones à faible rayon de confiance ou à forte hétérogénéité spatiale, l'organisation d'actions collectives pilotes pourra être visée en priorité (à l'image de l'ORAC en pays Sancerre-Sologne, qui réussit parce qu'elle n'implique pas un fort degré de confiance, étant très bien encadrée réglementairement).

Cela implique que la mise en œuvre des politiques territoriales devrait être à la fois plus adaptée à chaque situation et (paradoxalement) plus encadrée par la puissance publique. Cette dernière devant veiller à ce que les zones à fort rayon de confiance puissent exprimer leur potentiel auto-organisateur, mais aussi à ce que ce processus endogène ne se fasse pas au détriment des territoires " résiduels ». Là où le potentiel auto-organisateur est faible, la priorité devrait être mise sur les actions visant à élever et homogénéiser le rayon de confiance. Comme exemple typique de l'utilité d'un pilotage " par le haut », il est utile de comparer la carte de l'intercommunalité des départements voisins de l'Allier et du Puy-de-Dôme. Le premier est entièrement couvert par des communautés de communes et d'agglomération d'une taille appréciable (plus grande que les cantons), grâce à une politique volontariste du Conseil Général et du Préfet de département. Â l'inverse, le Puy-de-Dôme (où se situe le pays des Combrailles) comporte de nombreux vides (dont quelques enclaves), et de toutes petites communautés de communes « résiduelles » (à l'image de la figure 3), dont l'efficacité est de toute évidence limitée.

Ainsi, l'approche territoriale ne doit pas être considérée comme une panacée. S'il s'agit d'une notion qui a pu utilement mobiliser les énergies en faveur du développement local, elle doit être maniée avec précaution dans l'élaboration des stratégies de développement. Une intervention venant " du haut » peut notamment s'avérer utile pour corriger certaines inefficacités ou «inéquités " liées aux organisations spontanées d'acteurs. En outre, une vision trop « organique » ou « organiciste » des 
Les limites du territoire

territoires peut aboutir à faire croire qu'il est possible d'agir en faveur du développement en se dispensant de connaître à fond les mécanismes économiques à l'œuvre dans chaque secteur.

Cette étude théorique et empirique appelle plusieurs types de prolongements. D'abord, la notion de rayon de confiance, qui apparaît féconde pour comprendre l'organisation endogène des territoires, n'a été évaluée ici que de manière indirecte. Des indicateurs quantitatifs systématiques seraient précieux, mais il ne semble pas aisé de les produire à l'aide des bases de données statistiques existantes. D'autre part, l'articulation entre organisations endogène et politiques nationales et communautaires mérite un approfondissement particulier, sur le plan tant positif (comment les politiques influent sur l'organisation des acteurs locaux) que normatif (comment éviter les inefficacités pointées par les résultats de cet article). Enfin, la compréhension des mécanismes qui modèlent le rayon de confiance est un champ de recherche très vaste, encore largement inexploré, mais dont l'intérêt pour les politiques territoriales est évident. À l'heure où l'approche territoriale est de plus en plus prisée, ces développements apparaissent particulièrement pertinents.

\section{Annexe 1 : Preuve de la proposition 1}

Supposons que $\left|r^{\prime}\right| \leq 1$ et considérons un groupe de cohésion $G$ maximal au sens de l'inclusion. On va montrer que si $x$ et $y \in G$, alors $[x, y] \subset G$. Par définition du rayon de confiance, les individus membres de $G$ font nécessairement confiance à ceux de $[x, y]$ (en effet, chaque membre de $G$ fait confiance à $x$ et à $y$, et donc fait confiance à tous ceux qui sont entre eux).

Il reste à montrer que tous les membres de $[x, y]$ font confiance aux membres de G. Considérons $z \in] x, y$ [. Notons la borne supérieure de G. On a alors. En effet, comme $G$ est un groupe de cohésion, pour tous $x, y \in G$, on a $r(x) \geq|y-x|$. On a donc également par prolongement, autrement dit tous les membres de $G$ font confiance à un agent en. Inversement, si l'on fixe $y$, en utilisant le fait que la fonction de confiance $r$ est une fonction continue, on a par prolongement, autrement dit, un agent en fait confiance à tout membre de G. Comme G est un groupe de cohésion maximal, on a nécessairement .

Comme, on a . Par l'inégalité des accroissements finis, $r(z) \geq r(x)-(z-x)$, puisque $r^{\prime} \geq-1$. On a donc, ce qui implique que $z$ fait confiance à tous ceux qui sont à sa droite dans $G$. Par symétrie, il fait confiance à tous les éléments de G. En conséquence $G$ est forcément connexe.

Montrons à présent que tout groupe maximal $[a, b]$ est tel que . D'abord, il est évident que $b-a \leq r(a)$ et $b-a \leq r(b)$ (sinon l'individu en $a$ ou en $b$ ne ferait pas confiance à tous les individus du segment), et donc .

Supposons alors que. Considérons dans un premier temps le cas où il n'y a pas de problème de bord (c'est-à-dire $a>0$ et $b<N)$. Sans perte de généralité, on suppose que $b-a<r(a) \leq r(b)$. Considérons alors $\varepsilon \in[0,(r(a)-b+a) / 2]$. On a $\varepsilon<(r$ 
$(a)-b+a) / 2$ soit $r(a)>b-a+2 \varepsilon$. Comme $\left|r^{\prime}\right| \leq 1$, on est assuré que $r(a-\varepsilon)>r$ (a) $-\varepsilon$ et donc que $r(a-\varepsilon)>b-a+\varepsilon$ : l'individu en $a-\varepsilon$ fait confiance à tous les individus du segment $[a, b]$, qui n'est donc pas maximal.

Considérons à présent le cas où une extrémité du segment est un bord et supposons sans perte de généralité que c'est l'extrémité droite (c'est-à-dire que $b=$ $N)$. Si $r(a)>r(b)$, alors de toute évidence $b-a=r(b)$ (en effet, si on avait $b-a<$ $r(b)$ on pourrait sans problème étendre le groupe de cohésion $[a, b]$ vers la gauche. Inversement, si $r(a) \leq r(b)$, on peut appliquer directement le raisonnement du paragraphe précédent (il n'y a pas de problème de bord en $a$ car on a supposé que ).

La troisième partie de la proposition est une conséquence triviale de la deuxième.

\section{Annexe 2 : Preuve de la proposition 2}

On construit une configuration d'équilibre de la manière suivante. On commence par sélectionner un groupe de cohésion $G_{1}$ maximal au sens de l'inclusion ( $s$ 'il y en a plusieurs, on en choisit un au hasard), en notant que ce groupe maximal n'est pas nécessairement d'un seul tenant, puisqu'il n'est pas imposé que $\left|r^{\prime}\right|<1$. On enlève alors $G_{1}$ de l'ensemble des agents et on répète l'opération sur l'ensemble restant $[0, N] \backslash G_{1}$, et ainsi de suite jusqu'à ce que chaque élément de $[0, N]$ ait été affecté à un groupe de cohésion.

L'opération s'arrête nécessairement après un nombre fini d'itérations car par hypothèse la fonction de confiance avait une borne inférieure strictement positive. En effet, tant qu'il existe des ensembles de taille supérieure à non couverts par un groupe de cohésion, le groupe de cohésion maximal choisi à chaque itération sera nécessairement de taille supérieure à $\alpha$. Mais comme l'espace $[0, N]$ est de longueur finie, au bout d'un certain nombre d'itérations, il ne restera plus que des groupes de taille inférieure à $\alpha$. À ce moment (et s'il reste encore des agents non affectés à un groupe), les itérations suivantes consisteront à choisir comme groupes de cohésion maximaux chacun des ensembles d'un seul tenant restants (qui sont en nombre fini), et ce par ordre de taille décroissante. Notons également que tous les groupes obtenus dans une telle configuration ne sons pas nécessairement des intervalles fermés.

On montre alors que la configuration $\left\{G_{1}, \ldots G_{n}\right\}$ ainsi construite est un équilibre. Si ce n'était pas le cas, il existerait un groupe de cohésion $G$ (qui peut éventuellement comprendre des éléments de plusieurs groupes de la configuration $\left\{G_{1}, \ldots G_{n}\right\}$ ) dont tous les membres gagneraient à dévier. Considérons au sein de cet ensemble $G$ les membres les plus " anciens » au sein de l'itération précédente, c'est-à-dire ceux qui appartiennent à l'ensemble d'indice le plus bas dans $\left\{G_{1}, \ldots G_{n}\right\}$, et notons $i$ l'indice correspondant. Au moment où le groupe $G_{i}$ s'est formé, il était le plus grand groupe de cohésion possible parmi un ensemble qui incluait (entre autres) tous les éléments de $G$. Ceci est contradictoire avec le fait que pour être un déviant crédible, le groupe $G$ doit être forcément de taille supérieure à $G_{i}$. Un tel groupe déviant ne peut donc exister et $\left\{G_{1}, \ldots G_{n}\right\}$ est un équilibre. 


\section{Annexe 3. L'indicateur de cohésion locale et les zones d'étude}

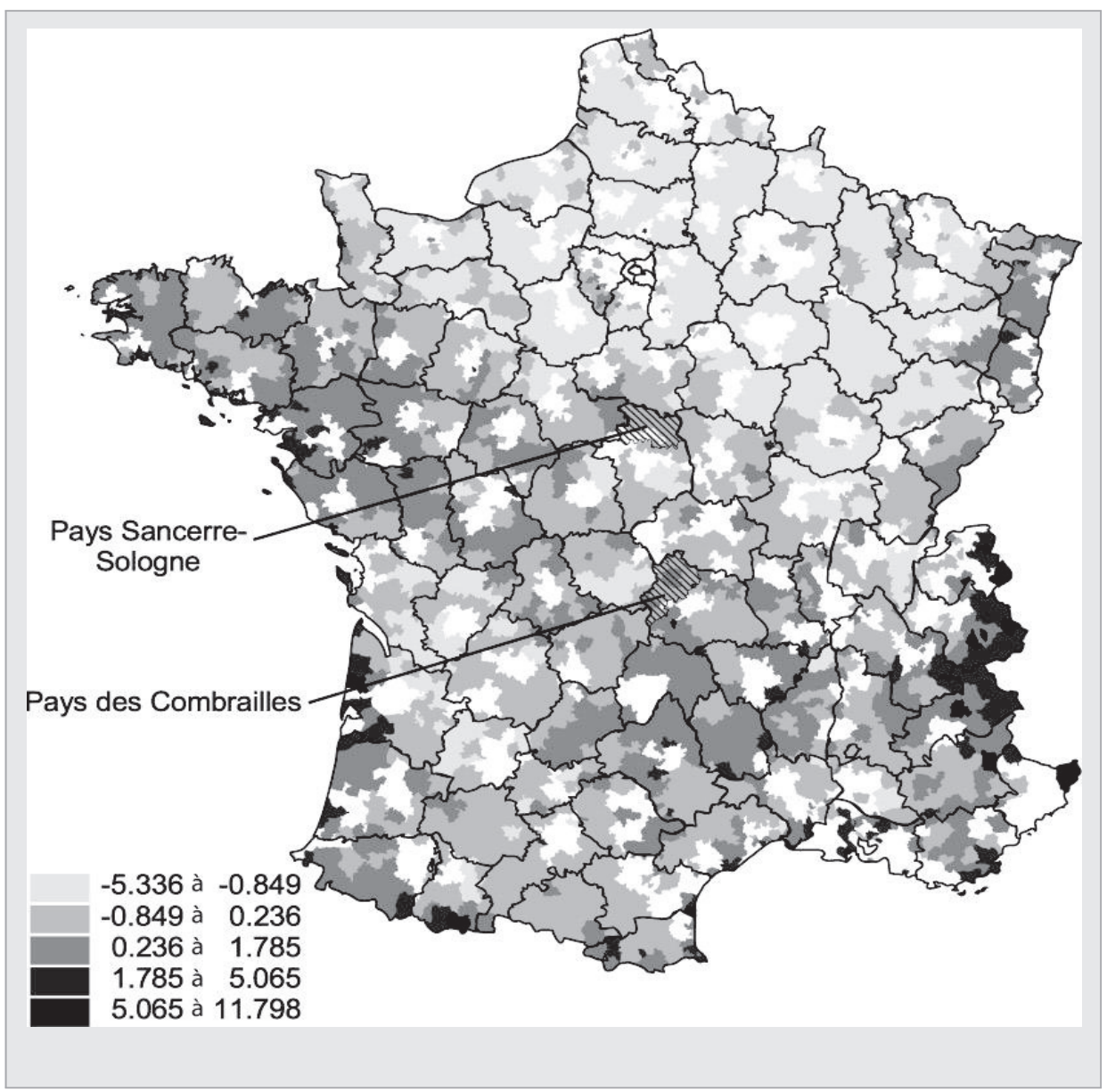

\section{Bibliographie}

AMIN A., 1993, "The globalization of the economy: an erosion of regional networks? ", in GRAHER G. (ed.), The embedded firm - On the socioeconomics of industrial networks, Routledge, London and New York, pp. 278-295.

CALLOIS J.-M., 2004, " Capital social et développement économique local. Pour une application aux espaces ruraux français », Revue d'Économie Régionale et Urbaine, $\mathrm{n}^{\circ} 4$, pp. 511-578.

CALLOIS J.-M., AUBERT F., 2007, «Towards indicators of social capital for regional development issues", Regional Studies.

CeLIMENE F., 1999, Le développement local : théories, pratiques et perspectives, Université Quisquya, Port au Prince.

COURLET C., 2001, «Les systèmes productifs localisés, un bilan de la littérature », Cahiers d'économie et de sociologie rurales, $\mathrm{n}^{\circ}$ 58-59, pp. 81-103. 
DiOn Y., LACOUR C., 2000, "La revanche du sectoriel et le renouveau de l'espace ", Revue canadienne des sciences régionales, vol. XXIII, $\mathrm{n}^{\circ} 2$, pp. 343-465.

FuKUYAma F., 1995, Trust: the social virtues and the creation of prosperity, Free Press, New York.

KREPS D.M., WilsON R., 1982, "Reputation and imperfect information ", Journal of Economic Theory, $\mathrm{n}^{\circ} 27$, pp. 253-279.

LAMARCHE R.H., 1995, "Local development : a selected review of literature ", Revue canadienne des sciences régionales, vol. XVIII, $\mathrm{n}^{\circ} 2$, pp. 235-254.

MAHE Ph., 2002, "Les expériences récentes de territorialisation des services des collectivités locales : enjeux et obstacles", Cahiers de la fonction publique et de $l^{\prime}$ administration, $\mathrm{n}^{\circ} 214$, pp. 6-9.

Maillat D., Crevoisier O., Lecoq B., 1991, "Réseau d'innovation et dynamique territoriale, essai de typologie", Revue d'Économie Régionale et Urbaine, $\mathrm{n}^{\circ} 3 / 4$, pp. 407-432

Pecqueur B., Zimmermann J.-B., 2004, Économie de proximités, Lavoisier, Paris.

PutNAM R.D., 1993, Making democracy work : civic traditions in modern Italy, Princeton university press, Princeton.

TODD E., 1990, L'invention de l'Europe, Le Seuil, coll. Points, Paris.

\section{Notes}

1 - Il existe peu de travaux qui tentent de modéliser l'émergence de territoires, bien que la théorie des coalitions, ou la littérature sur les effets d'agglomération, ainsi que les travaux d'économie évolutionniste sur les questions d'auto-organisation abordent des questions voisines. Cependant, ces travaux ne considèrent pas la question de la portée des relations sociales, qui est pourtant (implicitement) centrale dans l'idée de territoires auto-organisés.

2 - L'hypothèse de densité uniforme est assez inoffensive, à condition que l'on suppose cette densité bornée, notamment inférieurement par une valeur strictement positive (il faut alors remplacer la mesure naturelle de LEBESGUE par une mesure définie par la densité). Elle a été introduite uniquement pour alléger les démonstrations.

3 - La notion de confiance a été amplement développée par ailleurs dans la littérature économique et fait l'objet de débats complexes sur sa nature profonde et notamment la distinction importante entre une composante stratégique ( trust») et une composante liée au manque d'information sur les capacités des individus ("confidence "). L'émergence de la confiance dans les relations économiques a fait l'objet de nombreux travaux de théorie des jeux (notamment ceux de KREPS et WiLSON (1982) sur les conditions d'émergence de la coopération dans des situations de dilemme du prisonnier). Dans cet article, le choix a été fait d'éluder la dimension stratégique individuelle de la confiance pour se concentrer sur l'étude de l'émergence d'une organisation collective en fonction d'une propension plus ou moins grande des individus à coopérer avec leurs voisins.

4 - Cette représentation de type « tout ou rien » est bien entendu fruste, car il est clair que la confiance décroît de manière plus "douce » à mesure que l'on s'éloigne dans l'espace géographique ou social (par exemple, je peux prêter les clés de mon domicile à mon frère, mais pas à tous mes amis, auxquels néanmoins j'accepterais de prêter quelques centaines d'euros, etc.). La représentation choisie permet néanmoins d'obtenir des résultats suffisamment riches pour justifier une telle approximation.

5 - On n'examine donc pas la possibilité de mécanismes de compensation internes au groupe déviant. 
JOBNAME: No Job Name PAGE: 20 SESS: 15 OUTPUT: Mon Dec 17 15:57:17 2007

/juridique/acolin-RERU/444863m/05/01_callois

Les limites du territoire

6 - Cet indicateur est construit par agrégation (par analyse en composantes principales) de plusieurs mesures de sociabilité telles que: densité d'associations, densité de lieux de socialisation (installations culturelles et sportives, cafés), dons caritatifs, part d'abonnés au téléphone ayant leur nom sur l'annuaire (indicateur de confiance), intensité de coopération en agriculture.

7 - Début 2005, il existait 268 pays reconnus, et 73 en projet.

8 - Sources : recensement agricole de 1970, recensement de la population 1999, CD Rom Revenus des ménages 2001 de l'INSEE. 